\title{
Modification of the gamma-ray spectra from active galaxies by soft radiation of transiting luminous stars
}

\author{
Wlodek Bednarek ${ }^{a, *}$ and Julian Sitarek ${ }^{a}$ \\ ${ }^{a}$ Department of Astrophysics, Faculty of Physics and Applied Informatics, University of Lodz, Poland \\ E-mail: wlodek.bednarek@uni.lodz.pl, julian.sitarek@uni.lodz.pl
}

\begin{abstract}
Gamma-ray emission in active galaxies is expected to originate in a close priximity of the supermassive black hole surrounded by a reach cluster of luminous stars. We consider the effects of luminous stars crossing accidentally the gamma-ray beam close to the observer's line of sight. We show that soft radiation of massive stars can create enough target for transient absorption of the gamma rays in multi-GeV to $\mathrm{TeV}$ energy range. Time-dependent effects on the gamma-ray spectra due to the encounter with stars are investigated. As an example, we consider such effects on the spectra observed from a typical blazar, 1ES 1959+650 (in an active state) and also in the case of a radio galaxy M87 (in a low state). Observation of such transient characteristic features in the gamma-ray spectra of blazars and radio galaxies lays within the sensitivity of the future Cherenkov Telescope Array.
\end{abstract}

$37^{\text {th }}$ International Cosmic Ray Conference (ICRC 2021)

July 12 th - 23rd, 2021

Online - Berlin, Germany

\footnotetext{
${ }^{*}$ Presenter
} 


\section{Introduction}

It is supposed that high-energy $\gamma$ rays from active galaxies are produced within the inner jet or in the magnetosphere of the supermassive black hole (SMBH) due to their extremely short variability time scale. In fact, the $\mathrm{TeV} \gamma$-ray emission from blazars can change in the time scales as short as a few minutes (e.g. Mrk 501, see Albert et al. 2007; PKS 2155-304, see Aharonian et al. 2007 or IC 310, see Aleksic et al. 2014), This fast variability suggests that the emission site has to be close to the SMBH. Collimated $\gamma$-ray emission (a $\gamma$-ray beam) has to propagate through the surrounding region of the SMBH in which many compact objects are expected. The angular extend of such a beam in the blazar type of active galaxies, $\alpha$, is believed to be determined by the Lorentz factor of the inner jet which for $\gamma_{\mathrm{b}} \sim 10$ is $\alpha \sim 1 / \gamma_{\mathrm{b}} \sim 0.1 \mathrm{rad}$. In the case of $\mathrm{TeV} \gamma$-ray radio galaxies, the beams have to be much broader since the inclination angles of such galaxies, in respect to the observer's line of sight, are typically much larger than $~ 0.1$ rad, e.g. in Cen A (Tingay et al. 2001, Müller et al. 2014), NGC 1275: Vermeulen et al. 1994), and M87 (Biretta et al. 1999). The beam of energetic $\gamma$ rays has to encounter luminous stars that form a quasi-spherical halo around the central super-massive black hole (see Fig. 1).

We assume that the collimated beam of $\gamma$ rays is already produced in the close vicinity of SMBH. This $\gamma$-ray beam occasionally encounters compact luminous stars which pass close to the observer's line of sight. As a result, a transient, broad absorption feature is expected to appear in the continuum $\gamma$-ray spectrum due to the partial absorption of those $\gamma$ rays in the thermal radiation of the star, i.e. hardening of the spectrum below $\sim \mathrm{TeV}$ energies and a softening of $\gamma$-ray spectrum above $\sim 10 \mathrm{GeV}$. More details of this work can be found in Bednarek \& Sitarek (2021).

\section{Massive stars/relativistic jet encounter model}

We assume that the $\gamma$-ray production region in the vicinity of the SMBH (either the inner jet or the black hole magnetosphere) is surrounded by a young massive cluster of stars (see Fig. 1). The $\gamma$-ray spectra from active galaxies are at first order well described by a simple power-law, or a log-parabola function through the GeV-TeV energy range. It is likely that some luminous stars pass close to the line of sight of a distant observer with a given impact parameter $D$, defined as the shortest distance between the line of sight and the centre of the star. During such passage, the dense radiation field of the star can partly absorb $\gamma$ rays in a specific energy range.

The typical parameters of the early type luminous stars are: the surface temperature $T_{\star} \sim$ $3 \times 10^{4} T_{4.5} \mathrm{~K}$ and the stellar radius $R_{\star} \sim 10^{12} R_{12} \mathrm{~cm}$. The optical depth is the largest for the $\gamma$-ray photons with energies, $E_{\gamma} \sim 2 m_{\mathrm{e}}^{2} / \varepsilon \sim 67 / T_{4.5} \mathrm{GeV}$ in the case of head on collisions. It is $\tau_{\gamma-\gamma}(r) \sim D n_{\mathrm{ph}} \sigma_{\gamma-\gamma} \sim 110 R_{12} T_{4.5}^{3} / r$, where $\sigma_{\gamma-\gamma}$ is the cross section for $e^{ \pm}$pair production in collision of two photons, $n_{\mathrm{ph}}$ is the density of stellar photons and $r=D / R_{\star}$. This means that $\gamma$ rays moving even at some distances from the stellar surface can be absorbed.

The duration of the absorption effect on the $\gamma$-ray spectrum can be estimated for the known velocity of the luminous star on a circular orbit around the supermassive black hole. This velocity depends on the distance, $R=1 R_{1} \mathrm{pc}$, of the star from the SMBH. It is given by, $v_{\star} \approx 9.4 \times$ $10^{7}\left(M_{8} / R_{1}\right)^{1 / 2} \mathrm{~cm} \mathrm{~s}^{-1}$, where $M_{\mathrm{SMBH}}=10^{8} M_{8} M_{\odot}$ is the mass of the SMBH. The minimum time scale of the stellar transit is $\sim D / v_{\star} \sim 3 r R_{12}\left(R_{1} / M_{8}\right)^{1 / 2}$ hours. We conclude that the $\gamma$-ray 

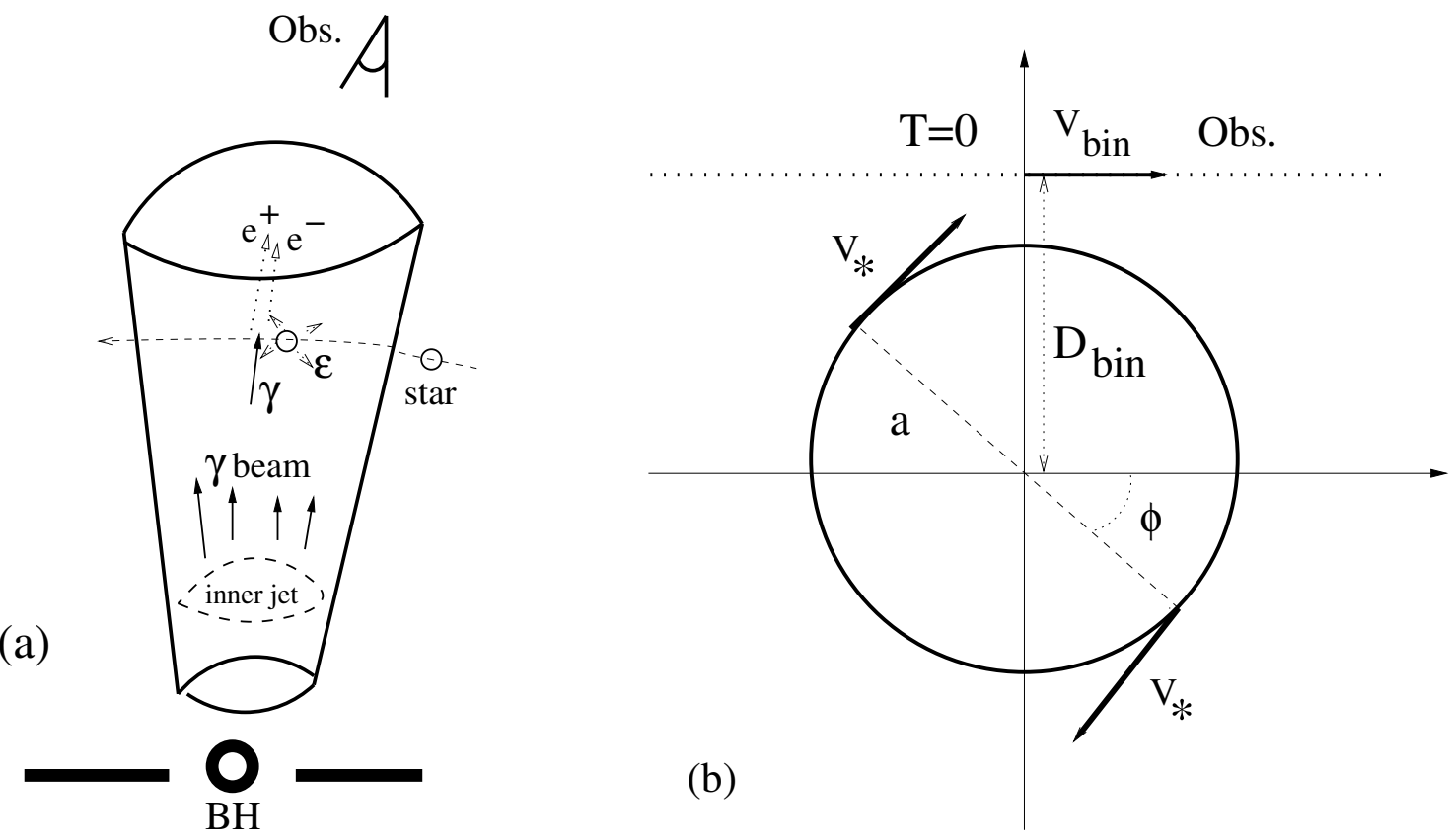

Figure 1: Figure (a): Schematic representation of the considered scenario for the production of specific absorption feature in the spectrum of GeV-TeV emitting active galaxies. A $\gamma$-ray beam is produced in the inner part of the jet. It meets on its way the soft radiation of a luminous star which entered the jet. $\gamma$ rays passing close by to the star are absorbed in soft radiation $\left(\gamma+\varepsilon \rightarrow e^{+} e^{-}\right)$. As a result the absorption feature should appear in the broad band $\gamma$-ray spectrum produced in the inner jet. Figure (b): The passage of a binary system through the $\gamma$-ray beam with the impact parameter $D$ in the reference frame of the binary system centre of mass. The observer (Obs.) moves with a velocity $v_{\text {bin }}$ along a straight line (dotted). The binary system contains two equal mass stars which move with velocity $v_{\star}$ on a circular orbit with the radius $a$. The orbit of the binary system lays in the plane perpendicular to the direction of the observer. The phase of the stars is marked by $\phi$. The time is measured from the closest distance between the observer and the centre of the binary system. (reproduced from Bednarek \& Sitarek 2021)

spectrum observed from active galaxies can be sporadically significantly modified in the multi-GeV to sub-TeV $\gamma$-ray energy range.

\section{Effects due to passage of a binary system}

The absorption effect of the $\gamma$-ray spectrum in the radiation field of the star can be evaluated by introducing the so-called "reduction factor" $(R F)$ which determines the ratio of integral $\gamma$-ray photon fluxes in specific range of energies $E_{\min }-E_{\max }$ : with the effect of the absorption to the non-absorbed (intrinsic) one. The $R F$ factor is defined as,

$$
R F_{E_{\min }}=\frac{\int_{E_{\min }}^{E_{\max }}\left(d N_{\gamma} / d E_{\gamma}\right) e^{-\tau} d E_{\gamma}}{\int_{E_{\min }}^{E_{\max }}\left(d N_{\gamma} / d E_{\gamma}\right) d E_{\gamma}} .
$$

We assume a differential $\gamma$-ray spectrum of a power-law type with an index of -2 , and the maximum energy at which the absorption effect of $\gamma$ rays is still important. $\tau$ is the optical depth for $\gamma$ rays in the radiation field of the star. 
Since half of the stars is expected to form binary systems, we consider the passage of luminous binary stellar system close to the observer's line of sight. In such a case, the effect of absorption of primary beam of $\gamma$ rays can have much more complicated time dependence since the distances between the observer's line of sight and each of the stars is additionally modulated by the movement of stars within the binary system (for the schematic geometry see Fig. 1). For simplicity, we consider that the binary system contains two stars of equal mass. Then, the velocities of specific stars within the binary system are $v_{\star}=\left(G M_{\star} / 4 a\right)^{1 / 2} \approx 4.1 \times 10^{6}\left(M_{1} / a_{13}\right)^{1 / 2} \mathrm{~cm} \mathrm{~s}^{-1}$, where the radius of the binary system is $a=10^{13} a_{13} \mathrm{~cm}$, and the masses of stars are $M_{\star}=10 M_{1} \mathrm{M}_{\odot}$. In fact, the transit velocities of binary systems are limited by the condition of disruption of the binary system in the gravitational field of SMBH. We estimate the minimum distance of the binary system from the SMBH at which the tidal forces on stellar companions are balanced by gravitational force of companion stars on, $L \approx\left(16 M_{8} / M_{1}\right)^{1 / 3} a \approx 1.8 \times 10^{-3} a_{13}\left(M_{8} / M_{1}\right)^{1 / 3}$ pc.

The cumulative effect of absorption of $\gamma$ rays (expressed by the reduction factor $R F$ ) in the radiation of stars in the example transiting binary system are shown in Fig. 8 in Bednarek \& Sitarek (2021), for the case of two threshold energies $E_{\min }=30 \mathrm{GeV}$ (upper figures) and $300 \mathrm{GeV}$ (lower figures). The simplest binary system case, namely two identical stars with the orbital plane perpendicular to the direction of the $\gamma$-ray beam, is considered. We show the absorption effects for the case of four impact parameters, $D_{\text {bin }}=10^{13} \mathrm{~cm}, 5 \times 10^{12} \mathrm{~cm},-5 \times 10^{12} \mathrm{~cm}$, and $-10^{13} \mathrm{~cm}$, and the initial phase $\phi=0^{\circ}$. The negative distances of the impact parameters denote opposite rotation direction of the stars than marked in Fig. $1 b$.

The significant reduction of the $\gamma$-ray emission is predicted to occur on a time scale of a few tens of days. However, a strong reduction of the $\gamma$-ray flux, in the form of characteristic two strong absorption dips, is also expected on a time scale of a few days when the observer's line of sight comes close to the stars within the binary system.

We also calculate the effect of absorption on the spectrum of the $\gamma$-ray beam (see Fig. 9 in Bednarek \& Sitarek 2021), for the case of the example binary system considered above. Interesting dependence of the spectrum on the transiting time can be observed. The basic feature, softening of the multi-GeV part of the spectrum and hardening of the sub-TeV part of the spectrum, can appear regularly during the transition event close to the minimum approach of the observer's line of sight. For the considered transition event, the effects of absorption are so strong that the $\gamma$-ray flux of the $\gamma$-ray beam can be drastically reduced. It can easily fall below the sensitivity limits of the $\gamma$-ray telescopes in this energy range. Note that considered here effects should strongly depend on the geometry of the binary system in respect to the observer's line of sight which greatly enhances the possibility of different absorption effects. Here we consider only the simplest possible geometry of the binary system, i.e. it is composed of two equal-mass stars and its plane lays in the plane of the sky. However, in general, the plane of the binary system can be inclined at an arbitrary angle to the plane of the sky. Moreover, the binary system can be formed from two luminous stars which significantly differ in their basic parameters (stellar mass and temperature, binary radius). Therefore, we expect even more complicated structures in the light curve of the transition of binary stars through the $\gamma$-ray beam formed close to the central engine of active galaxy. 


\section{The case of $1 \mathrm{ES} 1959+650$ and M87}

As an example, we consider the absorption effects on the $\gamma$-ray spectra due to the transiting binary system through the $\gamma$-ray beam in the case of two well known active galaxies from which $\mathrm{GeV}-\mathrm{TeV} \gamma$-ray emission have been reported by the Fermi-LAT and Cherenkov telescopes. The first active galaxy is a BL Lac object: 1ES 1959+650. Recently it was detected in the high state by the Fermi-LAT in the multi-GeV energies and by MAGIC in sub-TeV energies. We include the effects of absorption of $\gamma$-ray spectra observed from 1ES 1959+650 in the case of transit of the binary system with likely binary system and transit parameters. The modifications of the $\gamma$-ray spectrum by the transiting binary system at a specific time before minimum distance between the centre of the binary system and the observer's line of sight are shown for $T=-20-0$ days. We also consider the effect of a transit of the binary system on the spectrum of the well known radio galaxy M87 detected in a low state. Expected modifications of the $\mathrm{GeV}$ to $\mathrm{TeV} \gamma$-ray spectrum are reported in Fig. 2.

We confront these modified $\gamma$-ray spectra with the sensitivities of the $\gamma$-ray ground-based and space instruments. We used the publicly available instrument response functions (IRF) prod3b-2 of the CTA. For the stronger source, 1ES1959+650 we use short term (30 min) sensitivity IRF, while for a weaker M87 we use the corresponding mid-term (5 hr) IRF. Moreover, for 1ES1959+650 we also take into account the absorption in the extragalactic background light. Using a given flux model, the collection area of the instrument, and its migration matrix, we determine the expected excess rates in each estimated energy bin. With such computed expected number of observed $\gamma$ rays, and the rate of background events obtained from CTA IRF we estimate the expected uncertainty of the reconstructed flux. We consider that the flux can be probed at a given energy if the expected uncertainty is below $50 \%$ of the flux (i.e. resulting with $>2 \sigma$ point) and the expected number of $\gamma$ rays in this energy bin is above 10. In the case of 1ES $1959+650$ it is clear that even very short $(\sim 30 \mathrm{~min})$ exposure can be used for very accurate probing of the source spectrum and reproducing the absorption feature with high details. In the case of the much weaker M87 emission, while the intrinsic spectrum can be reconstructed without the stellar absorption (the emission is at the border of differential sensitivity for $5 \mathrm{hr}$ exposures), the absorption in stellar radiation would render the emission undetectable close to the transit time.

We investigate if the softening of the $\gamma$-ray spectrum at energies of tens of $\mathrm{GeV}$ can be observed by Fermi-LAT. We used the publicly available Pass 8 collection area to judge the achieved performance for a 10-day exposure of the Fermi-LAT instrument. We find that even for a high flux level comparable to the 1ES 1959+650 high state, even without the absorption of the $\gamma$ rays in the stellar radiation field, the sensitivity of the instrument does not allow to significantly probe the emission of the source above $\sim 10 \mathrm{GeV}$, where most of the effect is expected. Nevertheless, simultaneous observations in $\mathrm{GeV}$ range by satellite experiment to the observations in the sub-TeV range by Cherenkov telescopes would be still strongly desirable, since they can be used to constrain intrinsic source variability.

It is concluded that the CTA should easily detect the broad absorption feature between $\sim 30$ $\mathrm{GeV}$ and $\sim 1 \mathrm{TeV}$ (i.e. steepening of the part of the spectrum at lower energies and its hardening at larger energies) even during short observations. For bright sources, such effects might be even detected already with the current generation of Cherenkov telescopes. 

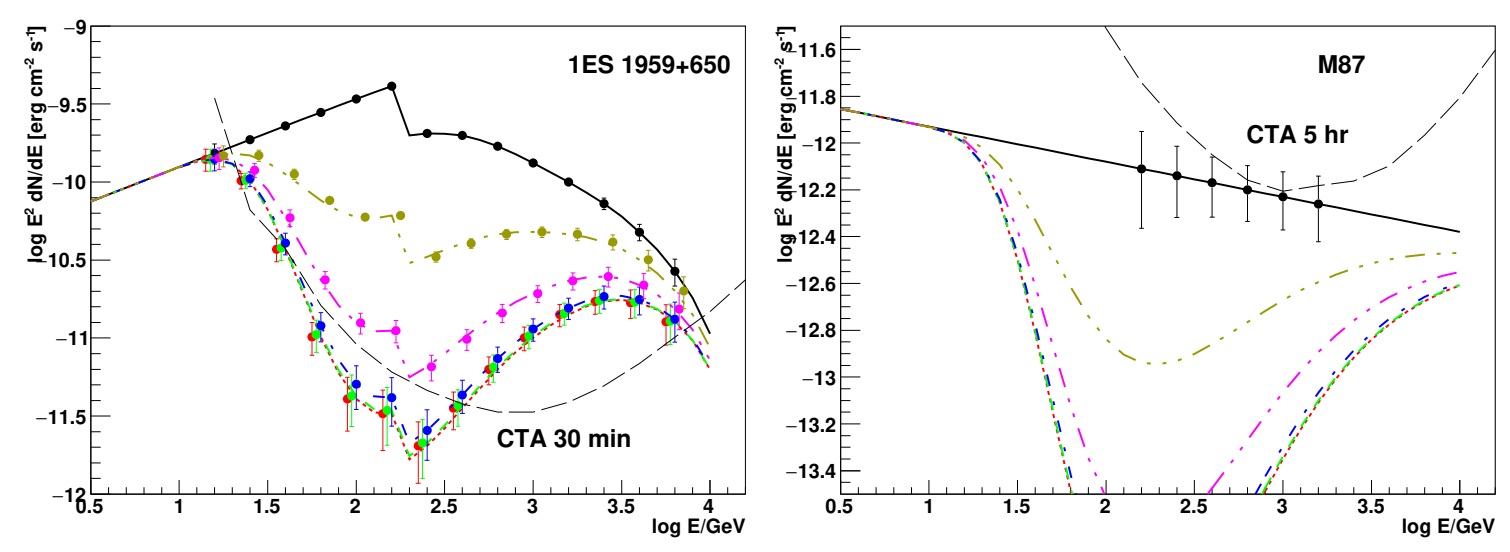

Figure 2: Effect of absorption on the $\gamma$-ray spectrum observed from two active galaxies: BL Lac 1ES 1959+650 (in the high emission state, see Acciari et al. 2020b, including the absorption in extragalactic background light using Dominguez et al. (2011) model) and the radio galaxy M87 (in the low state, see Acciari et al. 2020a). The absorption effect due to the passing binary system of stars with the parameters $R_{\star}=10^{12} \mathrm{~cm}, T_{\star}=3 \times 10^{4} \mathrm{~K}, a=10^{13} \mathrm{~cm}, D_{\text {bin }}=2 \times 10^{13} \mathrm{~cm}, v_{\star}=3 \times 10^{7} \mathrm{~cm} \mathrm{~s}^{-1}$, and $v_{\text {bin }}=3 \times 10^{7}$ $\mathrm{cm} \mathrm{s}^{-1}$ through the $\gamma$-ray beam is shown for 1ES $1959+650$ and M87 for the parameters, $R_{\star}=10^{12} \mathrm{~cm}$, $T_{\star}=3 \times 10^{4} \mathrm{~K}, a=10^{13} \mathrm{~cm}, D_{\text {bin }}=5 \times 10^{13} \mathrm{~cm}, v_{\star}=3 \times 10^{7} \mathrm{~cm} \mathrm{~s}^{-1}$. The spectra with absorption features correspond to different time measured in respect to the closest distance between the centre of the binary system and the direction to the observer: $\mathrm{T}=0$ days (dotted red curve), -2 days (dashed green), -5 days (dot-dashed blue), -10 days (dot-dot-dashed magenta), and -20 days (dot-dot-dot-dashed olive). The spectra are confronted with the $0.5 \mathrm{hr}$ (for 1ES 1959+650) or $5 \mathrm{hr}$ (for M87) sensitivity of CTA (see text). Points and uncertainties show the expected range and accuracy of the reconstructed spectrum by CTA for the given observation time with CTA (the points are slightly shifted between each curve for clarity). (reproduced from Bednarek \& Sitarek 2021)

\section{Discussion and Conclusion}

We considered the effects of transition of luminous stars through the $\gamma$-ray beam produced in the direct surrounding of the SMBH, i.e. either in the SMBH magnetosphere or in the inner part of the jet. It is assumed that from time to time stars pass close to the line of sight of a distant observer. If the transition is closer than hundreds of stellar radii of a luminous star, the $\gamma$ rays from primary $\gamma$-ray beam are partially absorbed. As a result, a broad dip at multi-GeV to sub-TeV range should appear in the continuous $\gamma$-ray spectral energy distribution produced around SMBH. This dip should be observed by the Cherenkov telescopes as a significant steepening of the spectrum above $\sim 10 \mathrm{GeV}$ and as a hardening of the spectrum below $\sim \mathrm{TeV}$ energies. Such a combined feature is characteristic, and hence can be easily disentangled from a possible intrinsic variability of the $\gamma$-ray beam. The time scale of such a transition event is predicted to take typically a few to a few tens of days. Therefore, it is expected that such events might be detected with the help of the current and future Cherenkov telescopes.

As an example, we apply our calculations to two well known active galaxies. In the case of the BL Lac type active galaxy 1ES 1959+650, we show that in its high state the effect of $\gamma$-ray absorption can be easily observed by the CTA telescopes (under construction) in the sub-TeV energy range during a few days even with very short nightly exposures. With enough exposure, CTA would be 
able to observe a clear hardening of the $\gamma$-ray spectrum at sub-TeV energies. We also investigate the case of radio galaxy M87 in the low emission state. In the case of the much weaker M87 emission, while the intrinsic spectrum can be reconstructed without the stellar absorption (the emission is at the border of differential sensitivity for $5 \mathrm{hr}$ exposures), the absorption in stellar radiation would render the emission undetectable close to the transit time.

In fact, for bright sources detection of such an absorption feature might be even possible with the present generation of Cherenkov telescopes (H.E.S.S., MAGIC and VERITAS) which sensitivity is nearly an order of magnitude lower than CTA. Unfortunately, in neither of the two simulated cases Fermi-LAT is sensitive enough to detect such absorption feature.

Detection of such transiting events (or their lack) should provide interesting constraints on the parameters of the central stellar clusters in active galaxies and on the production site of the $\gamma$-ray emission in active galaxies. Note that the duration of transition depends on the velocity of transiting stars which depends on the distance from SMBH, provided that its mass is known. On the other hand, the frequency of detected transiting events should allow us to constrain the distribution function of luminous stars in the central stellar cluster. The temperature of the star in turn affects strongly the depth of the dip in the spectral energy distribution and its position. In the case of transiting binary systems of two luminous stars, the time structure of transition should have curious features containing double peak structure with very fast change of the absorption efficiency. Observation of such double peaked absorption structures will allow us to constrain the surviving frequency of binary stellar systems in the direct vicinity of the SMBH.

Considered above absorption features can also appear at about an order of magnitude larger energies ( $\mathrm{TeV} \gamma$-ray energy range) in the case of transiting red hyper- and super-giants. Their typical radii and surface temperatures are $R_{\mathrm{RG}} \sim 10^{3} \mathrm{R}_{\odot}$ and $T_{\mathrm{RG}} \sim 3000 \mathrm{~K}$. Then, the optical depth becomes of the order of unity already at the distance from the star $D \sim 10 R_{\mathrm{RG}}$. In fact, large number of red giants is expected around SMHBs. For example, in the case of Cen A the number of red giants around SMBH is estimated on $\sim 10^{6}$ (see Wykes et al. 2014). The $\gamma$-ray emission of blazars is detected at the angle a factor of a few larger than the intrinsic opening angle of the jet (e.g. Pushkarev et al. 2009). Therefore, red giants can transit outskirts of such a $\gamma$-ray beam, avoiding disruption by the jet pressure (as considered by e.g. Barkov et al. 2010).

\section{Acknowledgments}

This work is supported by the grant through the Polish National Research Centre No. 2019/33/B/ST9/01904. This research has made use of the CTA instrument response functions provided by the CTA Consortium and Observatory, see https://www . cta-observatory.org/science/cta-performance/ (version prod3b-v2) for more details.

\section{References}

[1] Aharonian, F. et al. 2007 ApJ 664, L71

[2] Albert J. et al. 2007, ApJ 669, 862

[3] Aleksić, J., Ansoldi, S., Antonelli, L.A. et al. 2014 Science, 346, 1080A 
[4] Barkov, M.V., Aharonian, F.A., Bosch-Ramon, V. 2010, ApJ, 724, 1517

[5] Bednarek, W., Sitarek, J. 2021 MNRAS 503, 2423

[6] Biretta, J.A., Parks, W.B.S., Acchetto, F.M. 1999 ApJ 520, 621

[7] Domínguez, A., Primack, J. R., Rosario, D. J., et al. 2011, MNRAS, 410, 2556

[8] Müller, C. et al. 2014, A\&A 569, 115

[9] Pushkarev, A.B., Kovalev, Y.Y., Lister, M.L., Savolainen, T. 2009 A\& A 507, L33

[10] Tingay, S.J., Preston, R.A., Jauncey, D.L. 2001 AJ 122, 1697

[11] Vermeulen, R.C., Readhead, A.C.S., Backer, D.C. 1994 ApJ 430, 41

[12] Wykes, S., Hardcastle, M.J., Karakas, A.I., Vink, J.S. 2014, MNRAS, 442, 2867 